\title{
Mode Changes of a Planar 3 DOF Redundantly Actuated Parallel Robot
}

\author{
Takashi Harada
}

\begin{abstract}
A novel redundantly actuated planar parallel robot which enlarges the workspace by mode changes is proposed. Redundantly actuation and asymmetric design enables singularity avoided mode changes with loss redundancy but maintain non-singularity. In the assembly mode change, where the parallel robot transfers its configurations between two solutions of the direct kinematics, path planning which avoids singularity is proposed. In the working mode change, where the parallel robot transfers its configurations between two solutions of the inverse kinematics, the asymmetrical design contributes to singularity-free mode change. The singularities avoided mode changes were performed by newly developed prototype.
\end{abstract}

Index Terms - Prallel mechanisms, mechanical design, mode changes, workspace, singularity.

\section{INTRODUCTION}

The parallel robot has excellent mechanical features such as high accuracy, high rigidity, and small mass of the moving parts. However, mechanical interferences and singular configurations restrict the workspace. In order to expand the workspace, mode change of the parallel robot has attracted a lot of attentions [1], [2]. The parallel robot has multiple solutions of the direct and inverse kinematics. Each solution gives different configuration of the robot. By changing configurations from one solution to other, the parallel robot can expand its workspace. In the assembly mode change, the parallel robot changes configurations from one direct kinematics solution to other. In the working mode change, the robot changes those of inverse kinematics solutions. Conventional researches about the mode change have focused on non-redundant parallel robot. However, non-redundant parallel robot encounters a singular configuration during the mode changes. Redundantly actuation [3] assists the parallel robot avoiding the singular configuration during the mode change. However, there have been no researches about the mode change of redundant parallel robot, because of the complexity in design and control.

In this paper, a novel redundantly actuated planar parallel robot with a singularity-free mode change is proposed. Firstly, design and kinematics of the parallel robot are introduced. Secondly, the assembly mode change and working mode change of the parallel robot are discussed in detail. Then,

Manuscript received March 27, 2015; revised June 12, 2015. This work was supported by MEXT-supported program for the strategic Research Foundation to Private Universities 2012-2014, and JSPS KAKENHI Grant-in-Aid for Scientific Research (C) Grant Number 24560314.

T. Harada is with Department of mechanical engineering, Faculty of Science and Engineering, Kinki University, 3-4-1 Kowakae, Higashiosaka, 577-8502 Osaka, Japan (e-mail: harada@ mech.kindai.ac.jp). experimental results are shared by the prototype of the robot.

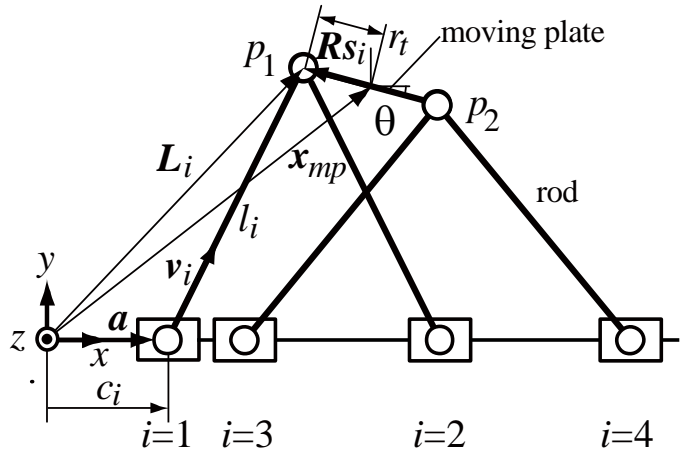

(a). 2-PRRR $\underline{P}$ parallel robot.

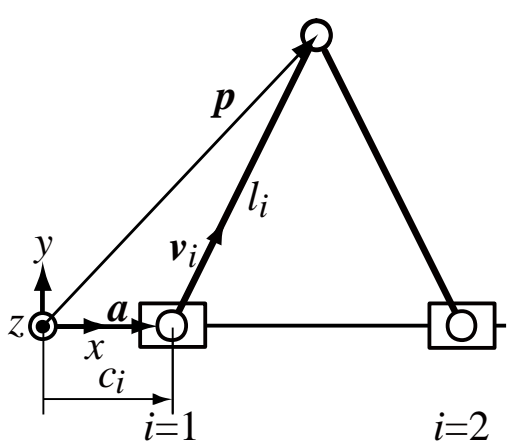

(b). PRRRP sub mechanism.

$\boldsymbol{x}_{m p}$ : position of the moving plate (MP)

$\theta$ : orientation of the MP

$p_{i}$ : position of $i^{\text {th }}$ pair on the MP

$r_{t}$ : half length of the moving plate

$\boldsymbol{a}:$ direction vector of the linear motor

$c_{i}$ : displacement of the motor

$c_{i}$ : displacement of the motor

$l_{i}:$ length of the rod

Fig. 1. 2-PRRRP parallel robot and PRRRP sub mechanism.

\section{2-PRRR $\underline{P}$ PLANAR 3 DOF PARALLEL ROBOT}

Fig. 1 (a) illustrates the 2-PRRR $\underline{P}$ ( $\underline{P}$ : active prismatic pair, $\mathrm{R}$ : passive rotational pair) parallel robot. The parallel robot has 3 degrees-of-freedom, its moving plate (MP) moves on the $x y$ plane and rotates around the $z$ axis. The moving plate is driven by 4 linear motors located at the prismatic pairs. Prototype of the robot is depicted in Fig. 2. The design of the robot is similar to the conventional 2-PRRR $\underline{P}$ parallel robot ARCHI [4], the moving plate of the robot enables unlimited rotation. In addition, our robot is adopted the multi-drive system, two linear motors drive on the same stator, enables large workspace along the directions of linear motors ( $x$ axis) [5]. Moreover, the robot is designed to achieve the assembly mode change, namely, it moves up and down passing through the hollow space on the base plate as shown in Fig. 3 (a). That 
novel design expands the workspace along the y direction. Asymmetrical design of the lengths of rods enables the working mode change as shown in Fig. 3 (b). This robot is applicable for the table mechanism of multi-axes machine tools.

\section{DiRect Kinematics And Assembly Mode Change}

Relationship between position $x_{m p}=[x, y]^{T}$ and orientation $\theta$ of the MP, and location of $i^{\text {th }}(i=1, \ldots, 4)$ linear motor $q_{i}$ is described by the loop closure equation [6] as,

$$
\boldsymbol{x}_{m p}+\boldsymbol{R}(\theta) s_{i}=\boldsymbol{L}_{i}=c_{i} \boldsymbol{a}+l_{i} \boldsymbol{v}_{i}
$$

$\boldsymbol{R}(\theta)$ represents the $2 \times 2$ rotational matrix.

The Jacobian matrices $\boldsymbol{J}_{x}$ and $\boldsymbol{J}_{c}$ of the parallel mechanism are given from Eq. (1).

$$
\begin{aligned}
& \boldsymbol{J}_{x} \dot{\boldsymbol{x}}=\boldsymbol{J}_{c} \dot{\boldsymbol{c}} \\
& \dot{\boldsymbol{x}}=[\dot{x}, \dot{y}, \dot{\theta}]^{T}, \dot{\boldsymbol{c}}=\left[\dot{c}_{1}, \ldots, \dot{c}_{4}\right]^{T}
\end{aligned}
$$

For the direct kinematics, locations of the linear motors are given, and the position and orientation of the MP are to be found. The direct kinematics of a parallel mechanism are solved by nonlinear simultaneous equations which express relationships between the position and orientation of the MP and displacements of the motors. Each solution of the direct kinematics gives the different assembly mode of the parallel mechanism. In general, it is difficult to solve the nonlinear simultaneous equations in closed analytical forms. The difficulty depends on the structure of the parallel mechanism. The structure of our proposed parallel mechanism, two rods are connected to the same rotational pair on the MP, enables it easy to solve the direct kinematics. The two rods, the rotational pair and two linear actuators is regarded as $\underline{P R R R} \underline{P}$ sub mechanism as shown in Fig. 1 (b). The solutions of the 2-PRRR $\underline{P}$ parallel mechanisms are given by the combinations of the solution of the PRRR $\underline{P}$ sub mechanisms. Moreover, kinematics analysis using the sub mechanisms gives the way how to achieve the singularity free assembly mode change.

Each position of the left and right pair of the MP is described as $\boldsymbol{p}_{j}=\left[p_{j}, q_{j}\right]^{T}(j=L, R)$. Each $\boldsymbol{p}_{j}$ corresponds to the output position of each sub mechanism. Position $\boldsymbol{x}$ and orientation $\theta$ of MP are simply given from the position of the pairs $\boldsymbol{p}_{j}$ as,

$$
\boldsymbol{x}=\frac{1}{2}\left(\boldsymbol{p}_{L}+\boldsymbol{p}_{R}\right), \theta=\tan ^{-1}\left(\frac{q_{R}-q_{L}}{p_{R}-p_{L}}\right) .
$$

Location of the linear motor is re-described $c_{j 1}$ and $c_{j 2}$.

$$
\left\{\begin{array}{l}
\left(c_{j 1}-p_{j}\right)^{2}+q_{j}{ }^{2}=l_{j 1}{ }^{2} \\
\left(c_{j 2}-p_{j}\right)^{2}+q_{j}{ }^{2}=l_{j 2}{ }^{2}
\end{array}\right.
$$

The direct kinematics of the sub mechanisms are given by the solution of Eq. (4). From the geometrical point of view, the simultaneous equations (4) represent the intersections of two circles on $x y$ plane. Central positions of the circles lie on the $x$ axis of the base coordinate frame. Radius and $x$ coordinate of each circle corresponds to length of the rod $l_{j i}$ and the actuator location $c_{j i}$, respectively. When the two circles has two different intersections, i.e., distance between the central positions of the two circles is shorter than the sum of the two lengths of the rods, the parallel mechanism has two different direct kinematic solutions given by one set of the actuators locations. This situation is depicted in Fig. 4 (a) and (b). The PRRRP sub mechanism has two assembly modes when the simultaneous equations (4) have two different solutions. Because the linear actuators of the sub mechanism align into the same line, the two configurations of the assembly modes are axisymmetric to the line of the actuators. The hollow space on the base and the interference-free mechanical design of the joint and link admit the mode change by avoiding the mechanical interference. In this paper, states of the assembly mode of the PRRR $\underline{P}$ sub mechanism are described $[+]$ and $[-]$ when the end point position of $y$ coordinate exists in the positive and negative area, respectively. When the simultaneous equations (4) has one solution (duplicate root), i.e., distance between the central positions of the two circles is the same length as the sum of the two lengths of the rods, two circles touch each other at the point of contact. This situation corresponds to the direct kinematics singular configuration of the parallel mechanism as shown in Fig. 4 (c). This state is described as [0]. Note here when the PRRRP sub mechanism changes the assembly mode, it encounters the direct kinematics singularity. When the distance between the central positions of the two circles is longer than the sum of the two lengths of the rods, the simultaneous equations (4) are impossible. The parallel mechanism has no direct kinematic solutions.
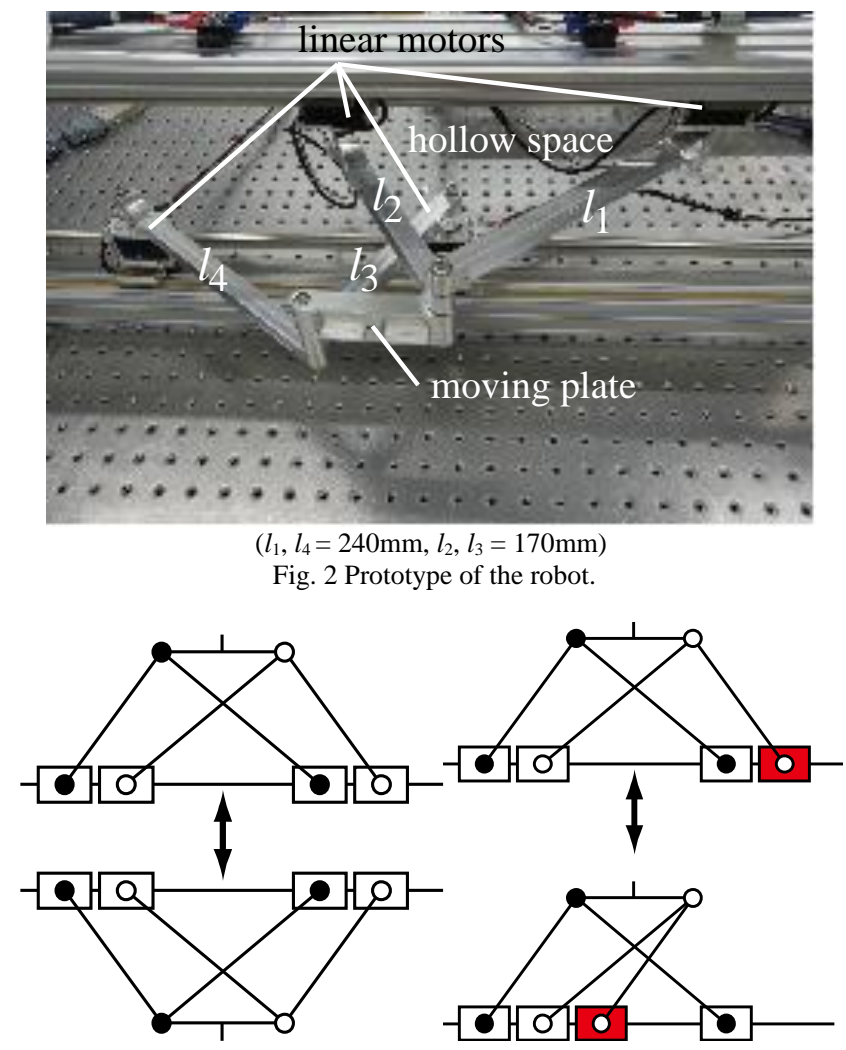

(a). assembly mode change (b). working mode change Fig. 3. Mode changes of the parallel robot. 


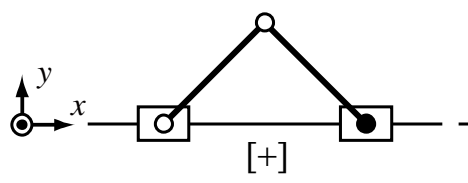

(a)

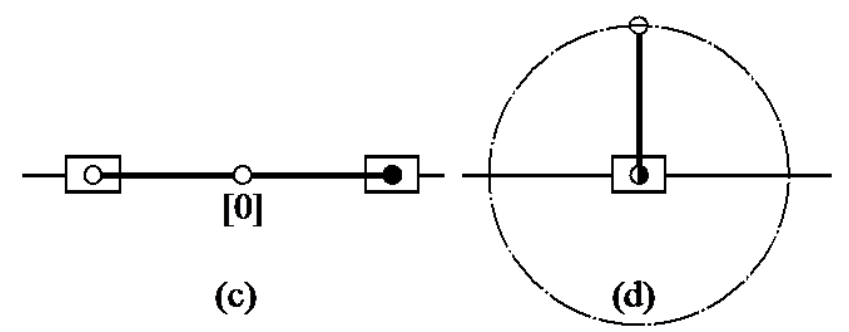

Fig. 4. Assembly mode of the PRRRP sub mechanism.

There still remains one state that the simultaneous equations become indefinite, when the two circles of Eq. (4) are identical. This situation corresponds to the complex kinematics singularity [6], as not every parallel mechanism admits it. Indeed, this singularity depends on the robot architecture. For the $\underline{P R R R} \underline{P}$ sub mechanism, this singularity occurs when the two actuators share the same location under the condition that the lengths of two rods are identical. We will adopt asymmetrical design that the two rods have different length for avoiding the complex singularity.

Assembly modes of the 2-PRRRP parallel robot are given as the combinations of the assembly modes of the PRRRP sub mechanisms. The parallel robot has 9 assembly modes as $[++],[+-],[+0],[-+],[--],[-0],[0+],[0-]$ and $[00]$. The state transition diagram of the assembly modes of the 2-PRRRP parallel robot is shown in Fig. 5. When the assembly modes of the 2-PRRR $\underline{P}$ parallel robot are $[++]$, $[+-],[-+]$ and $[--]$, rank of the Jacobian matrix $\boldsymbol{J}_{x}$ in Eq. (2) is 3 , i.e., the robot is not singular. At that time the parallel robot has end effector redundancy. When the assembly modes of the robot are [+ 0], $[-0],[0+]$ and $[0-]$, rank of the Jacobian matrix $\boldsymbol{J}_{x}$ is still 3 . The robot keeps the end effector non-singularity but loses the redundancy [7]. When the assembly mode of the robot is [0 0], rank of the Jacobian matrix $\boldsymbol{J}_{x}$ is less than 3, the robot is singular. Singularity-free assembly mode change from $[++]$ to $[--]$, or from $[--]$ to $[++]$, is achieved by tracing the route on the state diagram without passing the $\left[\begin{array}{ll}0 & 0\end{array}\right]$ mode as shown in Fig.6

\section{INVERSE KINEMATICS AND WORKING MODE CHANGE}

For the inverse kinematics, position and orientation of the MP are given, locations of the linear actuators are to be found.

Equation (1) is transformed as,

$$
\left(\boldsymbol{L}_{i}-c_{i} \boldsymbol{a}\right)^{T}\left(\boldsymbol{L}_{i}-c_{i} \boldsymbol{a}\right)=l_{i}^{2}
$$

The inverse kinematics of the parallel mechanism is given by solving the quadratic equation (5) with respect to $c_{i}$ as follows;

$$
c_{i}=\boldsymbol{L}_{i}^{T} \boldsymbol{a} \pm \sqrt{\left(\boldsymbol{L}_{i}^{T} \boldsymbol{a}\right)^{2}-\left(\boldsymbol{L}_{i}^{T} \boldsymbol{L}_{i}\right)+l_{i}^{2}} .
$$

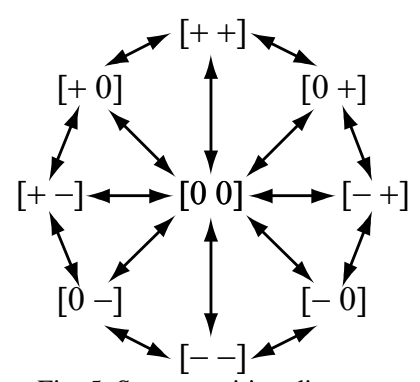

Fig. 5. State transition diagram.

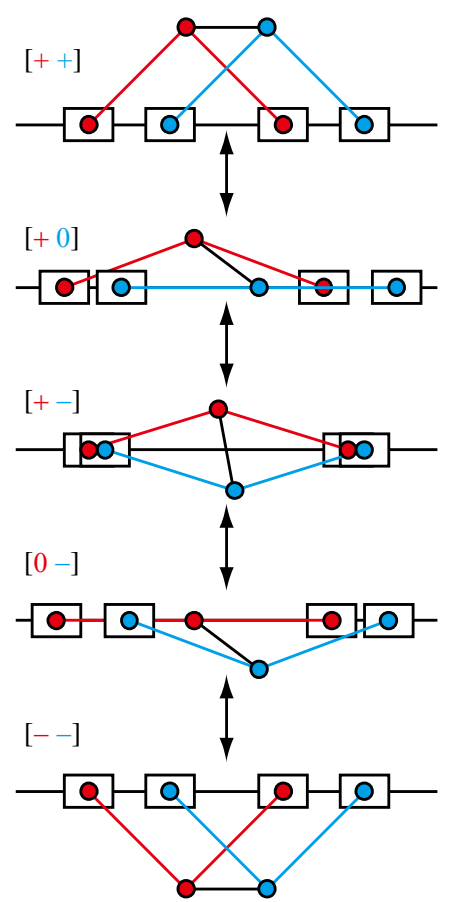

Fig. 6. Singularity free assembly mode change.

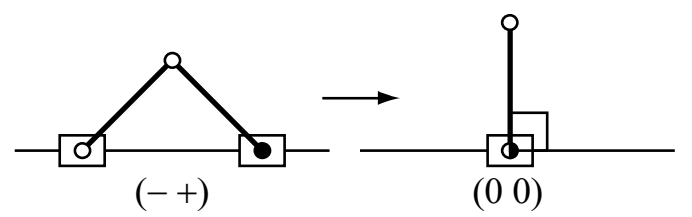

(a). symmetrical design.
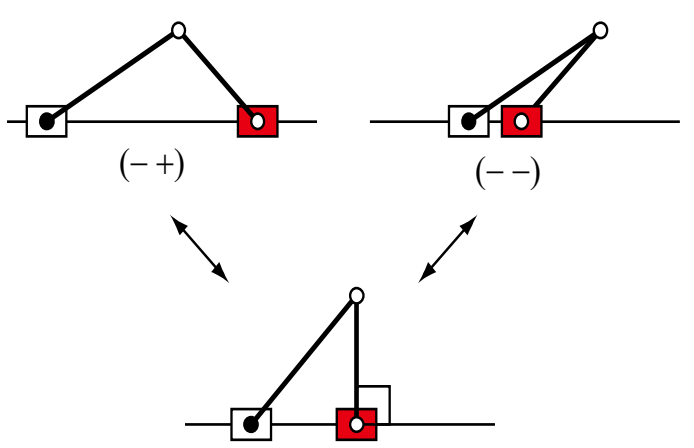

$(-0)$

(b). asymmetrical design.

Fig. 7. Working mode change of PRRRP sub mechanism.

Each $c_{i}$ has two solutions which are given by the signs before the square root are positive and negative, respectively. Corresponding configurations of the sub mechanisms are depicted in Fig. 7. In this paper, states of the working modes of the PRRRP sub mechanism are described (+) and (-) depending on the signs before the square root in Eq (6). Configuration of the link in (+) is axisymmetric to (-) with respect to the vertical line passing through the endpoint of the 
sub mechanism. When the term of inside the square root becomes zero, the quadratic equation (6) has a duplicated solution. This state in the inverse kinematics is described as (0) in this paper. At this state, direction of the rod is orthogonal to the direction of the linear actuator as shown in Fig. 7. This configuration of the parallel mechanism corresponds to the actuator singularity. Note here when the $\underline{\text { PRRRP }} \underline{\mathrm{P}}$ sub mechanism changes the working mode, it encounters the actuator kinematics singularity. In addition, when the lengths of the two rods of the sub mechanism are identical, the PRRRP sub mechanism falls into the complex kinematics singularity as shown in Fig. 7 (a). The asymmetrical design that the sub mechanism has different lengths of the rods contributes to the robot avoids the complex kinematics singularity as shown in Fig. 7 (b).

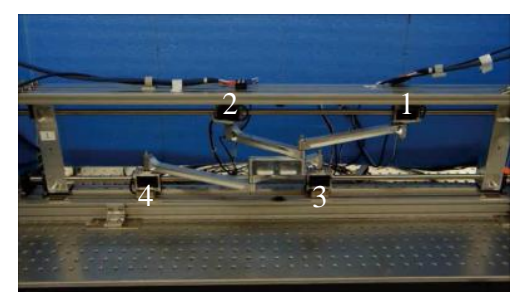

home configuration

assembly mode : $[++]$

working mode $:(-+-+)$

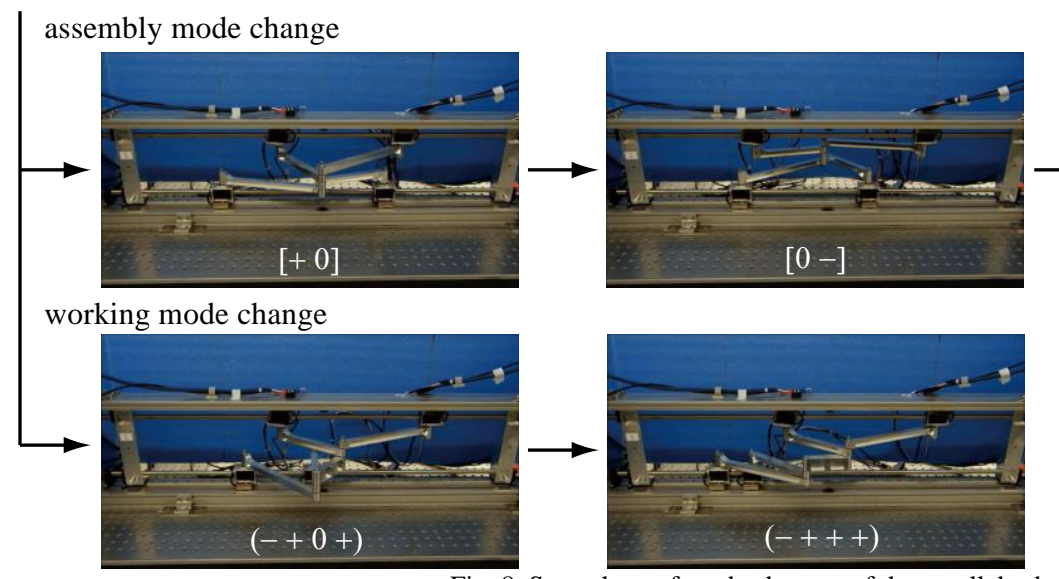

Fig. 8. Snap shots of mode changes of the parallel robot.

\section{EXPERIMENTS}

Fig. 8 illustrates snap shots of the assembly mode change and working mode change of the 2-PRRR $\underline{P}$ parallel robot. In the assembly mode change, the robot traces the route of $[++] \rightarrow[+0] \rightarrow[+-] \rightarrow[0-] \rightarrow[--]$. In the working mode change, the robot traces the route of $(-+-+) \rightarrow(-+0+) \rightarrow(-+++)$. While the mode changes, the PRRRP sum mechanism encounters singular configurations. At that time, the entire mechanism, 2-PRRR $\underline{P}$ parallel mechanism loses redundancy but maintains non-singularities.

\section{CONCLUSION}

In order to expand the workspace of the parallel robot, A novel 3dof redundantly actuated 2-PRRR $\underline{P}$ parallel robot with singularity-free mode change is proposed in this paper.

1) The state transition for the singularity free assembly mode is proposed by kinematics analysis using the PRRR $\underline{P}$ sub mechanisms.

2) The singularity free assembly mode change is achieved by the asymmetrical mechanical design.

3) The singularity free mode changes were performed by the newly developed prototype.

\section{REFERENCES}

[1] L. Campos, L. F. Bourbonnais, I. Bonev, and P. Bigras, "Development of a Five-bar parallel robot with large work -space," in Proc. ASME IDETC/CIE, vol. 2, pp. 917 922, 2010.
[2] C. Budde, M. Helm, P. Last, A. Raatz, and J. Hesselbach, "Configuration switching for workspace enlargement," Robotic Systems for Handling and Assembly, pp. 175-189, 2010.

[3] J. P. Merlet, "Redundant parallel manipulators," Laboratory Robotics Automation, vol. 8, pp. 17-24, 1996.

[4] F. Marquet, S. Krut, O. Company, and F. Pierrot, "ARCHI: a new redundant parallel mechanism-modeling, control and first results," in Proc. IEEE/RSJ IROS, 2001, pp.183-188.

[5] T. Harada and M. Nagase, "Impedance control of a redundantly actuated 3-DOF planar parallel link mechanism using direct drive linear motors," in Proc. IEEE ROBIO 2010, pp. 501-506.

[6] L. W. Tsai, Robot Analysis: The Mechanics of Serial and Parallel Manipulators, Wiley Interscience, 1999, pp. 116-168.

[7] T. Harada, "Design of a redundantly actuated asymmetric linear DELTA parallel mechanism for singularity-free mode changes," Applied Mechanics and Materials, vol. 575, pp. 711-715, 2014.

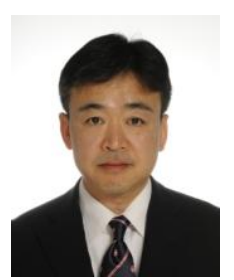

Takashi Harada received the B.S. degree and M.S. degree in industrial mechanical engineering from Osaka University in 1985 and 1987 . He received the $\mathrm{Ph} . \mathrm{D}$. degree in mechanical engineering from Osaka University in 2000.

He has been a professor at the Department of Mechanical Engineering, the Faculty of Science and Engineering at Kinki University, Japan, since 2006. He was a visiting professor at the Department of Mechanical Engineering at McGill University, Canada from 2012 to 2013. His main subject of research is precision mechanical engineering, specifically, robust measurement and control of mechanical systems in an actual environment. Recently, he has been focusing on the design and control of parallel mechanisms. He received the outstanding paper award in the Japan Society of Mechanical Engineers 2012, and the best conference paper award at IEEE ROBIO 2010. He is a member of JSME, RSJ, ASME and IEEE. 